\title{
Analysing the Curvature of the Pectoralis Muscle in Mammograms
}

\author{
Christina Olsén \\ Department of Computing Science, Ume a Unirersity, SE-901 87 Umeå, SWEDEN \\ colsen@cs.umu.se \\ http://www.cs.umu.se/ ${ }^{\sim}$ fredrikg/ic_iman.htm
}

\begin{abstract}
One of the most important criteria considering the assessment of the diagnostic quality in mammograms is the pectoralis muscle. In this paper a routine that automatically analysis the shape of the muscle and compute measurements needed to determine the quality concerning the muscle is presented. The method is based on a division of the $\mathrm{m}$ uscle in to sub images and using the Hough transform to compute the slope of each sub images line. The performance to determine the shape of the pectoralis muscle is $95.5 \%$ correctly classified mammograms based on 155 randomly chosen images from the MIAS database. Therefore, the conclusion is that the proposed shape analysis method is a reliable method for determining the shape of the muscle.
\end{abstract}

\section{Introduction}

Mammography is a standard breast imaging technique to detect breast cancer [7]. The technical requirements and the ability to judge the mammogram properly are stringent and a computerized system that assists the personnel within a mammography unit might ease some of the rigorous demands [6]. To enable a radiologist to make a proper judgment, the mammograms must be of sufficient quality [2]. Thus, the quality assurance will also be an important elemen $t$ in a computer-aided diagnosis system. There is a mental pressure for the patient to be recalled, due to inadequate mammograms, for another examination, but also the concern that there is cancer present. If the recall rate due to inadequacy in image quality can be reduced, it will be more cost-effective and more comfortable for the patient.

When properly obtained, the mediolateral oblique view (MLO) is the most important mammographic view since it best visualizes the tissue adjacent to the chest wall and the axillary tail, where the majority of cancers occur [4]. One of the most important criteria considering the assessment of the image quality is the pectoralis muscle. Since mammography can be a painful procedure for the $\mathrm{w}$ omen the muscle may be tense and this may result in an incorrect performed MLO view. A successfully performed MLO view (concerning only the pectoralis muscle) will visualize the pectoralis muscle with a convex anterior margin.

Fig. 1, shows four different shapes of the muscle commonly seen with MLO positioning [5]. In pattern 1, the pectoralis muscle is relaxed, properly mobilized and compression is sufficient. This is the ideal pattern, showing anterior 

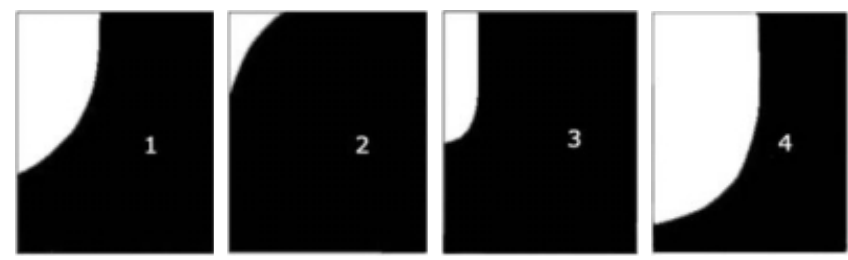

Fig. 1. Illustration of four patterns of pectoralis muscle commonly seen with MLO positioning. The figure is redrawn from Eklund et al., [5].

convexity. Pattern 2 shows a concave anterior margin. This is a consequence of that the pectoralis muscle was not properly mobilized medially and this is most likely caused by an unrelaxed muscle. Pattern 3 shows the anterior margin of the pectoralis muscle oriented parallel to posterior margin of the image, indicating an unsuccessful compression. Before compression is applied it is important to mobilize the muscle and breast medially allowing the muscle to move back laterally. Pattern 4 is an example of a well-developed and unusually mobile pectoralis muscle. If it is the pectoralis muscle rather then the glandular tissue that limits the compression, the tissue will not be sufficiently compressed.

\section{Previous work}

There is very little research done within the issue of automatic evaluating adequacy in mammograms. Chandrasekhar, [3] points out the importance of adequate mammograms. Not only because of the convenience for patients but also that inadequate mammograms might cause failure in algorithms in computeraided diagnosis systems.

Recently Chandrasekhar et al., [4] published a paper concerning an investigation of automatic determination of mammogram adequacy in MLO based on several criteria presented by Eklund et al., [5]. One of the criteria investigated is concerning the curvature of the pectoralis muscle. The method presented is based on approximating a straight line from the inferior and superior extent of a fitted curve and comparing the area of this triangle with the area enclosed by the image edges and a fitted curve of the pectoralis muscle [9]. There were some problems with the method caused by the inaccuracy of the fitted curve and according to Chandrasekhar et al., [4] the measurement is not a reliable estimate of the curvature of the anterior margin.

This paper concerns an alternative approach to evaluate the curvature of the pectoralis muscle. The algorithm is based on using the Hough transform to approximate a straight line representing the anterior edge of the muscle. The main idea of this approach is to divide the muscle into sub images representing the muscle at sequential different locations and then analyze each sub images slope of the lines found by the Hough transform. The proposed method in this paper is reliable and yields very good results. Since there is no presentation of 
any measurable values of the performance of the algorithm proposed by Chandrasekhar et al., [4] a quantitative comparison of the performance with our proposed algorithm is impossible. Clearly, since it is pointed out that the method by Chandrasekhar et al., is not reliable and our method is reliable at least in 95.5\% of the mammograms our method is superior to the method presented by Chandrasekhar et al., [4].

\section{Background of Algorithm}

All the images used in this implementation were taken from the MIAS database. This database is the standard database used within computer-aided mammographic research and it contains 322 images all taken with the MLO view representing 161 bilateral mammogram pairs. The mammograms in the database also contain some artefacts such as plastic tape and scratches. Even though the images are obtained with the MLO view, the pectoralis muscle is not always visible.

A cleaning algorithm has been implemented that minimize the image, removes the labels and some noise and removes the empty black columns/rows that contain no information. The images were thresholded with a global threshold and reduced in size to $20 \%(205 \times 205$ pixels $)$ of the original images $(1024$ $\times 1024$ pixels). For a more throughout description see [10].

\section{The Algorithm}

The initial step is to segment the pectoralis muscle from the cleaned mammogram by approximating the pectoralis muscle with a straight line and then approximating a polygon to represent the segmented object. This procedure needs to be carried out in order to estimate a measurement of the entire muscle. After the entire muscle is segmented the next step is to evaluate the shape of the muscle, where the measurement of the segmented muscle is used when dividing the muscle into 15 different slices, see Sect. 4.2.

\subsection{Finding initial estimate of the pectoralis muscle}

In order to locate the pectoralis muscle some assumptions has to be made. First, the knowledge of the fact that the database contains pairs of mammogram is used. Every odd numbered image is flipped so that all breast tissue is facing right. Secondly, when all the images are facing the same way it is assumed that the pectoralis muscle is located in the upper left corner of the mammogram.

The Hough transform The algorithm used to find the pectoralis muscle is based on work by Karssemeijer [8]. The main idea is to perform the Hough transform on a gradient image. In the implementation the $(\rho, \theta)$ line parameterisation is used: $\rho=x \cdot \cos \theta+y \cdot \sin \theta$, where the origin of the polar co-ordinate is at the 
centre of the image. The angles in the Hough transform were chosen to be $115^{\circ}-$ $165^{\circ}$, because of the assumption that the pectoralis muscle has certain degree of inclination.

Finding the suitable lines in the Hough-space By choosing all lines as: $L=l: g(\rho, \theta)>0.95 M$, were $M=\operatorname{argmax}_{(\rho, \theta)} g(\rho, \theta)$ several lines are found. To be able to find the lines that closest represent the line of the pectoralis muscle, the lines were grouped into several groups depending on how much one $\rho$ differs from the next and so on. If the difference between one $\rho$ and the next is larger than a value $\alpha$ then the line with larger $\rho$ is put into another group. The line with the largest $\rho$ within the group with the majority number of lines was chosen to be the line that absolutely closest represented the actual muscle.

\subsection{The shape of the pectoralis muscle}

The main idea of this approach is to divide the muscle into sub images representing the muscle at sequential different locations and then analyze each sub images slope of the lines found by the Hough transform.

Division of the muscle into slices In order to investigate the concavity or convexity of the muscle the pectoralis muscle was divided into 15 different slices. The size of each slice is depending on the height and width of the entire muscle, segmented as described in 4.1. Every slices width starts at the posterior border of the muscle. The first slice corners are $(0,0)$, (width, 0$)$, (0, height $/ 2)$, (width, height $/ 2$ ) then the width and height is reduced with $5 \%$ for each slice. The height starts at the superior border of the muscle and for every new slice the starting point has increased several pixels, see Fig. 4.

Computing the slope for each slice and create a code array The Hough transform presented above was performed on each slice. The angles of interest were chosen to $89^{\circ}-179^{\circ}$, to be able to detect all possible lines. From those lines the slopes were computed and put into a slope array. All the slopes depending on differences in the slope values were divided into different interval in a nonlinear fashion where each interval is represented with a number $0 \ldots 7$. All those numbers were put into a code array. Fig. 2 gives a visual description of the intervals. As seen in the figure there are three intervals $(3,4$ and 5$)$ that are larger then the rest of the intervals, the purpose of this representation is to visualise that the most common slopes in a mammogram fall into these intervals.

The code array was then analyzed by estimating the sign of the changes. If there is no change in the analyze array the pectoralis is said to be straight and for the pectoralis muscle to be either concave or convex, the difference between the maximum slope value and the minimum has to be a fixed value, $\beta$. This value was decided after have run through all images in randomly selected training set of the database and the mean value $m$ of all the differences were computed. $\beta$ is a fraction of $m, \beta=m / \lambda$, were $\lambda=\{1,2,4,6,8,10\}$ was tested. 


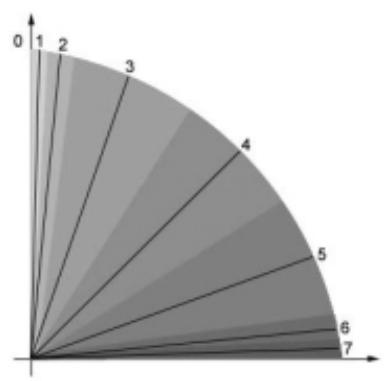

Fig. 2. An illustration of the intervals of the slope values.

Handling special cases The images contain disturbances such as, noise, scratches and tapes. These cause problems when computing the slope for each sub image. Therefore a routine was developed to handle unnatural changes. An unnatural change is changes in the code array that differ several numbers from one step to the next, which means an abrupt change in direction, see Fig 2. For instance, situations of changes were a code at position $i$ in the code array is 5 and the code at position $i+1$ is 2 is classified as unnatural changes.

\section{$5 \quad$ Result}

The MIAS database was divided randomly into two groups containing $161 \mathrm{im}-$ ages each. One of the groups, the training set, was used to compute all necessary parameters. The other group, the test set, was used for evaluation of the algorithm. After performing several tests on the training set all the parameters were determined:

- It was found that $\alpha=1.5$ (defined in Sect. 4.1) was a good threshold for the division into different groups.

- Two alternative ways of dividing the muscle into slices were tested on the training set of the images. These two alternative ways of division are further described in Sect. 5.1.

- The difference between the maximum and minimum slope value, $\beta$ was determined by computing the mean $m$ and by testing different $\lambda . \lambda=2$ gives the best result, see Sect. 4.2.

- The division of the slopes into different interval was established.

\subsection{Performance of the algorithm}

The proposed algorithm performs very well on most mammograms in the MIAS database. The algorithm was also tested on 20 mammograms from the South 

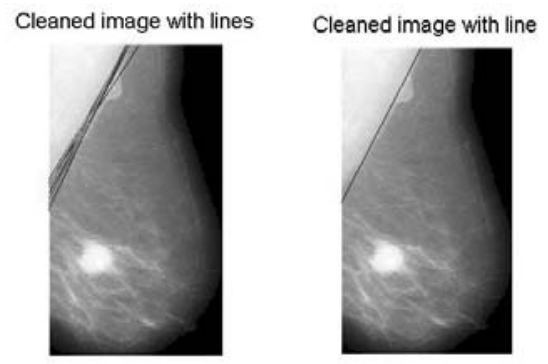

Fig. 3. Mammogram no. 28. a) With all candidate lines from the Hough-space plotted. b) The straight line representing the pectoralis muscle on a global level.

Table 1. An overview of the performenace of the algorithm

\begin{tabular}{|l|r|r|}
\hline Correctly classfied & 148 & $95.5 \%$ \\
Incorrectly classfied & 7 & $4.5 \%$ \\
\hline Total & 155 & $100.0 \%$ \\
\hline
\end{tabular}

Florida Database, [1] and all 20 mammograms were classified correctly, which indicates that the propose algorithm works satisfactory on a different database as well.

It seems like picking the line with the largest $\rho$, see Sect. 4.1 in the majority group is a very good method for finding the best line. The line chosen to be the line absolutely closest to the pectoralis muscle is very close to the line found by choosing the line as $M$, see Sect. 4.1. However, the method implemented seems to work better. The lines in the majority group found, by the Hough transform, represent the area closest to the edge of the muscle were most edge pixels are found, see Fig. 3. Then choosing the largest $\rho$ within that group gives the most anterior line.

The shape evaluating method, described in Sect. 4.2, proved to yield good results. Mammograms where incorrectness is caused by no or barely visible muscles are removed from this evaluation, since these images are not really candidates for the shape evaluation. The size of the entire muscle were approximated. If the area of the muscle is smaller then $3.5 \%$ of the cleaned breast image then the shape will not be evaluated, since it is too small to yield reliable measurements. In a complete quality evaluation system, this need to be handled before the system reaches the shape evaluation. As seen in Table 1, $95.5 \%$ of the 155 randomly chosen mammograms from the MIAS database was correctly classified.

The method chosen to divide the pectoralis muscle into 15 slices yields reliable results with good performance. Two alternative ways of division were tested on the training set of the images. In the first method, the slices height was fixed. Each height of the slices were set to the total height divided by $2,3, \ldots, 8$. The second alternative method tested was to keep the slices centred on the anterior edge of the muscle. This method turned out to be sensitive to noise and artefacts. 

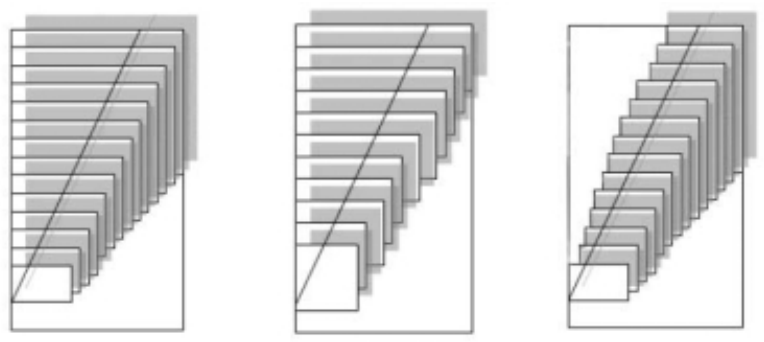

Fig. 4. a, b and c. Visual descriptions of the 15 slices. a) Shows the way the 15 slices is divided. b) and c) show alternative ways of division.

None of the two alternative methods worked as well as the one chosen. Fig. 4 gives a visual description of the ways of division.

The pectoralis muscle edge was found in each slice and a slope was computed. The analysis of the code array and division of the slopes into different intervals works well. For instance, considering mammogram no. 233 in Fig. 5a, which shows an convex anterior margin. The slopes in this mammogram are decreasing for each slice and the mammogram is correctly classified as convex. Mammogram no. 78 , on the other hand, shows a concave anterior margin, although at a closer observation it can be seen that the slopes are first increasing in several parts of the muscle and then decreasing at the end of the muscle. In other words the superior margin of the muscle is concave and the inferior margin is slightly convex, see Fig. 5b. Since the whole of the muscle is concave the mammogram is correctly classified as concave.

Problems occur when the pectoralis muscle is evenly concave and convex. Considering mammogram no. 135 in Fig. 5c. In this mammogram, the anterior margin of the muscle is concave at the beginning and then becomes convex at the end. This muscle should maybe be treated as it is concave because that is the shape criteria indicating inaccuracy. In the method implemented this is not always the case. Some of those muscles are therefore wrongly classified. The analysis of the code array works very well on most images but for $100 \%$ accurate performance of the algorithm it needs to be improved to be able to handle rare but important problem such as the one described.

An extension of this shape algorithm would be needed to decide whether or not the pectoralis muscle is parallel to the posterior border for a complete evaluation. A parallel muscle will be found in the range of about $89^{\circ}-100^{\circ}$.

\section{Conclusion}

We propose a method for extracting and determining the shape of the pectoralis muscle. The method is evaluated on a standard database and we conclude that the method is reliable and yields very good results. With some adjustments, 

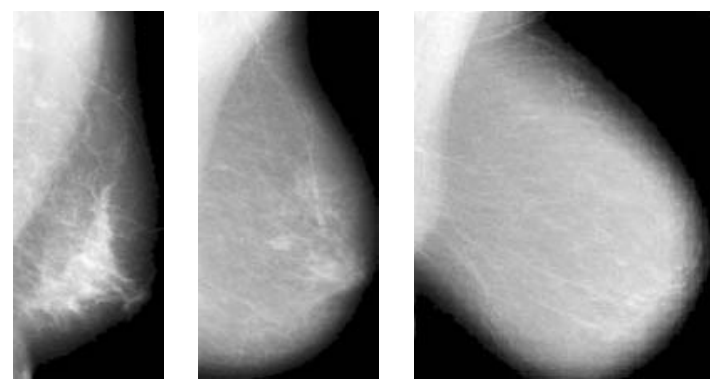

Fig. 5. Illustration of three mammograms from MIAS database a, b and c. a) Mammogram no. 233. b) Mammogram no. 78. c) Mammogram no. 135. The mammograms visualises some of the different shapes the pectoralis muscle may have: Convex, concave and mixed.

particularly regarding the analysis of the code array, the extraction of the shape is believed to produce even better results.

\section{References}

1. University of south florida digital mammography. Digital Database for Screening Mammography (DDSM) http://marathon.csee.usf.edu/Mammography/Database.html, August 2002.

2. S. Carlson. Personal communication with chief radiologist Stina Carlson at the University Hospital Umeå Sweden, February 2002.

3. R. Chandrasekhar. Systematic Segmentation of Mammograms. PhD thesis, University of Western Australia, Department of Electrical and Engineering, Nedlands, 1996.

4. R. Chandrasekhar, S.M. Kwok, and Y. Attikiozel. Automatic evaluation of mammographic adequacy and quality on the mediolateral oblique view. In proceedings of IWDM, pages 182-186, 2002.

5. G.W. Eklund, G. Cardenosa, and W. Parson. Assessing adequacy of mammographic image quality. Radiology., 190:297-307, 1994.

6. F. Georgsson. Algorithms and Techniques for Computer Aided Mammographic Screening. PhD thesis, UMINF-01.15 Umeå University, Department of Computing Science, 2001.

7. S. Heywang-Köbrunner, I. Schreer, and D. Dershaw. Diagnostic Breast Imaging. Thieme, Stuttgart, 1997.

8. N. Karssemeijer. Automated classification of parenchymal patterns in mammograms. Physics in Medicine and Biology., 43:365-378, 1998.

9. S.M. Kwok, R. Chandrasekhar, and Y. Attikiozel. Automatic pectoral muscle segmentation on mammograms by straight line estimation and cliff detection. In proceedings of ANZIISC, pages 67-72, 2001.

10. C. Olsén. Automatic determination of mammogram adequacy. Master's thesis, UMNAD 424/02 Umeå University, Department of Computing Science, 2002. 